ICNMM2006-96090

\title{
TURBULENT FLOW IN A MICRO-CHANNEL
}

\author{
Tomasz A. Kowalewski, Slawomir Blonski, Piotr M. Korczyk \\ Institute of Fundamental Technological Research, Polish Academy of Sciences, tkowale@ippt.gov.pl
}

\begin{abstract}
Turbulent flow of water in a narrow gap of an emulsifier is investigated experimentally using micro-PIV (micro Particle Image Velocimetry) technique and compared with numerical predictions performed using the commercial code Fluent. The purpose of the investigations is to develop a procedure for wellcontrolled generation of mono-disperse suspension of micro droplets. These droplets will form a matrix for collection of nano-particles into well-structured configuration [1]. The micro-flow measurements are based on epi-fluorescence illumination and high-speed imaging. The experimental data are compared with the numerical results obtained using both turbulent and laminar flow models. It was found that, due to small channel dimensions and very small flow development length, the turbulent energy dissipation takes place mainly in the gap and shortly behind it. Very low amount of oil-phase fraction in investigated emulsions justifies us to use mean energy dissipation estimated for pure water to predict mean diameter of oil droplets. These predictions are validated using experimental data for the emulsion.
\end{abstract}

\section{NOMENCLATURE}

Symbol Description

$Q_{V} \quad$ Volumetric flow rate

$Q_{M} \quad$ Mass flow rate

$V \quad$ Velocity

$\langle V\rangle \quad$ Mean value of velocity

$V^{\prime} \quad$ Fluctuation of velocity

Re Reynolds number

$d \quad$ Diameter

$\rho \quad$ Density

$\sigma \quad$ Interfacial tension

$p \quad$ Pressure

$\mu \quad$ Viscosity

TKE Turbulent Kinetic Energy

$S^{\|}$

$S^{\perp}$

$\varepsilon$

Unit
$\mathrm{m}^{3} / \mathrm{s}$
$\mathrm{kg} / \mathrm{m}^{3}$
$\mathrm{~m} / \mathrm{s}$
$\mathrm{m} / \mathrm{s}$
$\mathrm{m} / \mathrm{s}$
$\operatorname{dimensionless}$
$\mathrm{m}$
$\mathrm{kg} / \mathrm{m}^{3}$
$\mathrm{~N} / \mathrm{m}^{3}$
$\mathrm{~Pa}$
$\mathrm{Pas}$
$\mathrm{m}^{2} / \mathrm{s}^{2}$
$\mathrm{~m}^{2} / \mathrm{s}^{2}$
$\mathrm{~m}^{2} / \mathrm{s}^{2}$
$\mathrm{~m}^{3} / \mathrm{s}^{3}$

\section{INTRODUCTION}

The design of new procedures for fabrication of nanostructured materials is one of the "hot topics" in the contemporary materials science, due to the great potential for their application in various modern technologies. Preparation of micro and nano-size structures is a fundamental topic of research in this area. The experimental and numerical work is focused on production of emulsion droplets in turbulent flow using a narrow-gap homogeniser (Fig. 1). The need to get detailed and accurate measurements in micro-scale of the device enforces the application of new experimental techniques, unusual in classical fluid mechanics. One of them is micro-PIV, full field microscopic velocity measurement using tracers with dimension of only several nano-meters.

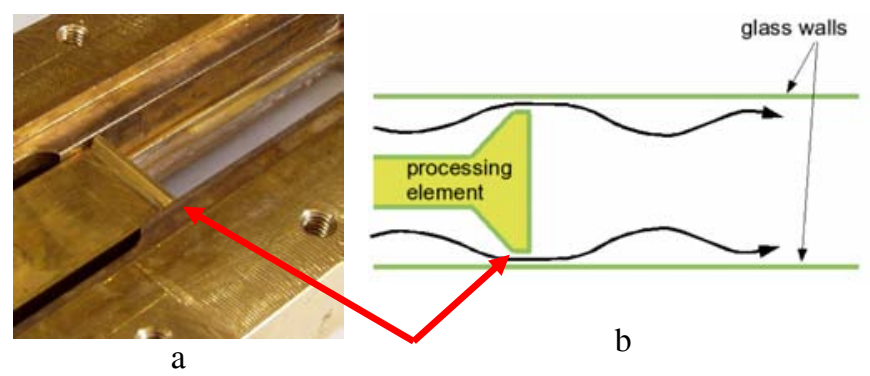

Figure 1: Model of the narrow-gap homogeniser: a) top view after removing covering glass wall, b) schematic crosssection; arrows point to the analysed micro-channel.

A simple model of shear flow induced emulsifier consists of a small channel formed between two glass plates, separated by a triangular obstacle (Fig. 1). Dimensions of the gap between glass plates and processing element (triangular obstacle) are $0.4 \mathrm{~mm}$ in height, $1 \mathrm{~mm}$ in length and $15 \mathrm{~mm}$ in width (comp. Fig. 2).

The flow rate used was $Q_{V}=0.204 \mathrm{dm}^{3} / \mathrm{s}$. A typical value of the velocity in the emulsifier is about $5 \mathrm{~m} / \mathrm{s}$ for the entrance region and $20 \mathrm{~m} / \mathrm{s}$ in the gaps, which corresponds to Reynolds number 7500 and 8000 , respectively. 
For investigation and visualization of production process of the emulsion, mainly for observation of oil-droplets breakup, high-speed imaging was used.

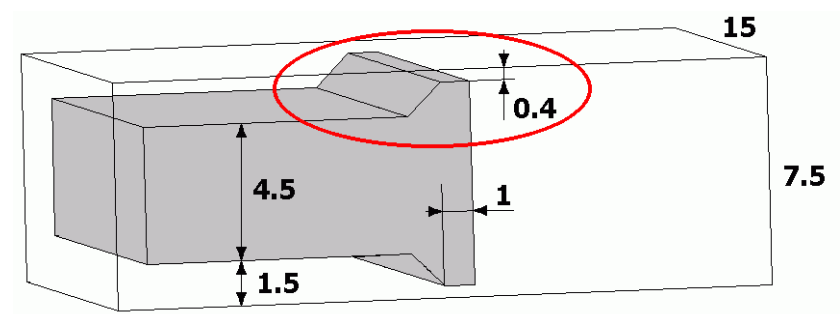

Figure 2: Geometry of the processing element of the emulsifier model and location of the analysed microchannel; micro-channel size: height $0.4 \mathrm{~mm}$, width $15 \mathrm{~mm}$ and length $1 \mathrm{~mm}$.

\section{EXPERIMENTAL SETUP AND PROCEDURE}

The main part of the experimental set-up consists of epifluorescence microscope, model of the emulsifier with transparent windows, light source and digital cameras (Fig. 3). A pressurized nitrogen vessel, attached to the working liquid supply bottle was used for flow acceleration. The gas pressure was stabilized at a selected level using the pressure compensation reservoir. Under applied gas pressure the liquid was driven through the homogeniser to the collecting bottle. The flow rate was set varying the reservoir pressure and using the system of valves. The exact value of the flow rate was obtained by measuring the time necessary to fill up a calibrated quantity in the collecting bottle.

The flow was investigated using pure water and very dilute water-oil emulsion. When the emulsion was used, the experiment was repeated several times by pouring liquid from the collecting bottle back to the supply bottle using a by-pass tube. It was commenced after "manual-shaking” of water-oil mixture prepared in the supply bottle. Usually 5 to 10 passes were used to record the development of the emulsion in the vicinity of the processing element.

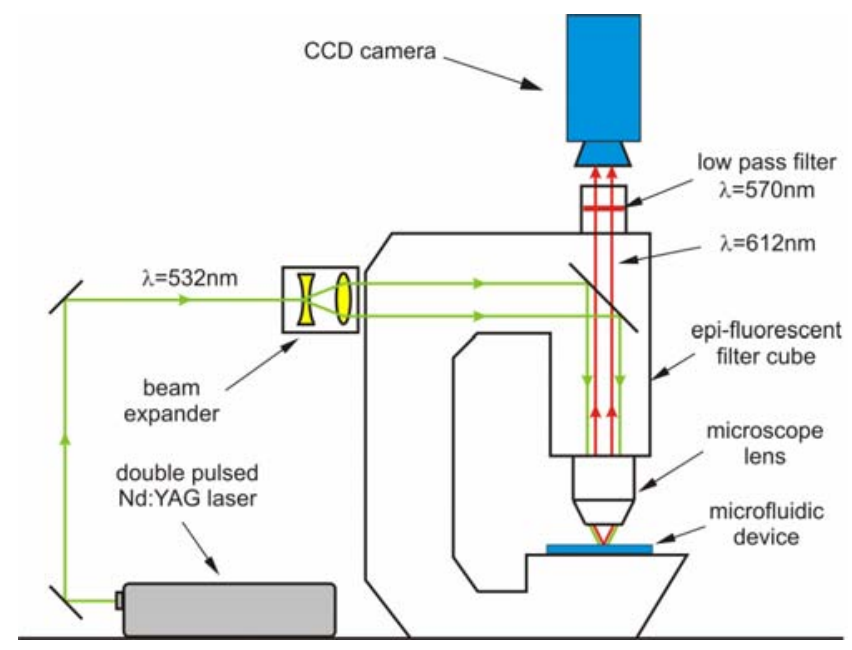

Figure 3: Scheme of the micro-PIV experimental set-up.
The flow was examined using epi-fluorescence microscope (Nikon Eclipse 50i). To obtain different magnification ratios four far field lenses were used: 4x (NA 0.13 / WD 17.1mm), 10x (NA 0.3 / WD 17.30mm), 20x (NA 0.35/ WD 24mm), and 50x (NA $0.45 /$ WD 17mm). The flow observed under the microscope is characterized by large relative displacements, therefore application of high-speed recording techniques becomes essential. In our case, the image width of 1280 pixels corresponds, for the highest magnification ratio, to the object dimension of $0.172 \mathrm{~mm}$. With flow velocity of $1 \mathrm{~m} / \mathrm{s}$ the illumination time necessary to freeze the motion appears to be less than 100ns. Such short illumination time can only be achieved by using pulsed laser light.

Three different light sources were tested: (i) built-in microscope halogen lamp with condenser for backlight illumination; (ii) CW 5W Argon laser (496nm+515nm) for epifluorescence and flow visualization; (iii) Nd:YAG $30 \mathrm{~mJ}$ (532nm) double pulsed laser used for epi-fluorescence flow velocity measurements (PIV) and as a 5ns short backlight illumination for emulsion imaging.

For the flow study two high-resolution (1280x1024pixels) 12 bit cameras were used. For the short exposure imaging the PCO SensiCam camera was used. When coupled with the double pulse laser it permitted acquisition of two images at the minimum time interval of 200ns and exposition time of $5 \mathrm{~ns}$. However, with this system, pairs of images can be registered at about $3.75 \mathrm{~Hz}$ repetition rate only. Hence, the second camera PCO1200hs was used for high-speed flow visualisation. This camera permits recording of full resolution images (1280x1024pixels) at 636fps, and at over 40kfps for decreased vertical image resolution. An electronic shutter of the camera allows to freeze relatively high-speed motion, it is attained at the cost of overall light intensity. With continuous illumination (halogen lamp, argon laser) the shortest viable illumination time using this camera appeared to be $1 \mathrm{~ms}$.

The flow structure measurements were performed for pure water seeded with fluorescent tracers. The micro-flow measurements based on epi-fluorescence illumination and highspeed imaging allowed for collecting detailed data on the shear stresses in the turbulent flow, necessary for modelling of the emulsification process. Thereafter, for the same flow conditions, the visualization of oil droplets in a very dilute emulsion was performed. It is assumed that flow structure is not affected by the presence of droplets and results obtained for the single phase are applicable for calculation of the droplets deformation.

\section{FLOW STRUCTURE MEASUREMENTS}

The flow structure study was based on micro-PIV technique. It permitted measurements of instantaneous twodimensional velocity fields for the selected sections of the channel. The micro-PIV measurements were performed using pure water in the simple model of a shear flow induced emulsifier. It consisted of a small channel formed between two glass plates separated by a triangular obstacle (Fig. 1b). Water was pumped under pressure through a small slit between the obstacle and side walls of the channel. Observations of the flow were performed through a transparent top or bottom wall. Flow was analysed in the vicinity of the channel centre defined by the vertical symmetry plane. 
The PIV velocity measurements were performed using seeding of fluorescent polystyrene spheres, $2 \mu \mathrm{m}$ in diameter (Duke Scientific Inc.). The particle volumetric concentration was very low $(<0.0001 \% \mathrm{wt})$, hence they did not affect the flow structure. Particle Image Velocimetry (PIV) [2,3] based on correlation of pairs of images was used to evaluate instantaneous velocity fields in the channel. These full field data allowed evaluation of local velocity gradients, hence for estimation of conditions for the droplet break-up [4].

Unlike typical PIV methods, micro-PIV does not utilize a thin laser sheet to illuminate the seeding particles. The whole investigated volume was flooded with the laser light using beam expander and the microscope objective (Fig. 3) [5]. Once the particles are exposed to 532nm light (green) from the laser, they emit red light with an emission maximum at $612 \mathrm{~nm}$, as specified by the supplier. The time-span in which particles continue to fluoresce after the laser pulse is of the order of nanoseconds, so motion induced blurring of the particles does not occur in the PIV images. Two low pass filters, mounted between the objective and the camera, permit only the fluorescent red light to pass, while preventing the green laser light to be detected by the camera. The PIV images present well detectable bright spots of the seeding particles. Only particles being within the depth of focus are recorded. Particles that are out of focus add background noise, limiting applicability of the technique to thin layers of fluid (max. 10-15mm). The flow was observed through the upper window.

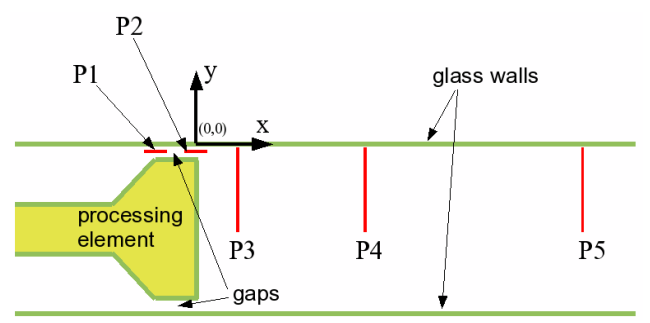

Figure 4: Schematic drawing of the emulsifier with the coordinate system and locations of measured velocity fields.

By traversing the field of observation in the horizontal and vertical direction, the position of the interrogated flow plane was selected. The vertical resolution depends on the depth of field of the objective. For the micro-PIV experiments performed using the $10 \mathrm{x}$ objective the vertical resolution was estimated to be $10 \mu \mathrm{m}$. The horizontal resolution of the velocity field measurements for this objective was $0.5 \mu \mathrm{m}$. The accuracy of the velocity measurement depends on several experimental factors (quality of the images, seeding concentration, particle displacement), as well as on the vector evaluation procedure. Using in house developed software and by evaluating uniform, known flow of water through the micro-channel, the error of velocity measurement was estimated to be below $5 \%$.

As results, five profiles of velocities were obtained from the PIV measurements - each of them in different locations (Fig. 4 and Table 1). Profiles at positions P1 and P2 were obtained along the $x$-direction in the vicinity of the entrance to the gap and the end of the gap, respectively. Profiles at positions P3, P4 and P5 were obtained along the y-direction $1 \mathrm{~mm}, 3 \mathrm{~mm}$ and $8 \mathrm{~mm}$ behind the gap, respectively. For profiles at positions P1 and P2 measurements of the velocity field were repeated several hundred times, and no time-fluctuations were observed. Flow seems to be laminar here.

Table 1: Profiles span for selected locations

\begin{tabular}{ccc}
\hline Profile location & $\begin{array}{c}\mathrm{X} \\
{[\mathrm{mm}]}\end{array}$ & $\begin{array}{c}\mathrm{Y} \\
{[\mathrm{mm}]}\end{array}$ \\
\hline \hline P1 & from -1.45 to -0.7 & -0.2 \\
P2 & from -0.35 to -0.35 & -0.2 \\
P3 & 1 & from 0 to -3.75 \\
P4 & 3 & from 0 to -3.75 \\
P5 & 8 & from 0 to -3.75 \\
\hline
\end{tabular}

Velocity of the flow rapidly increases in the vicinity of the entrance to the gap, from $8 \mathrm{~m} / \mathrm{s}$ to $16 \mathrm{~m} / \mathrm{s}$ (Fig. 5), and then, just behind the gap, rapidly decreases from $18 \mathrm{~m} / \mathrm{s}$ to $16 \mathrm{~m} / \mathrm{s}$ (Fig. 6).

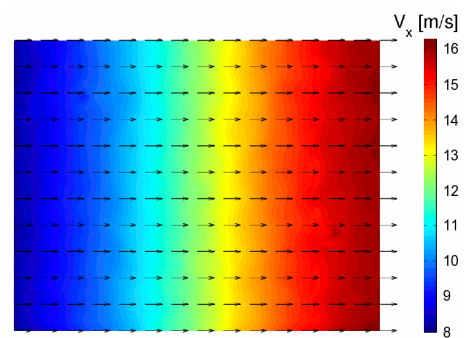

(a)

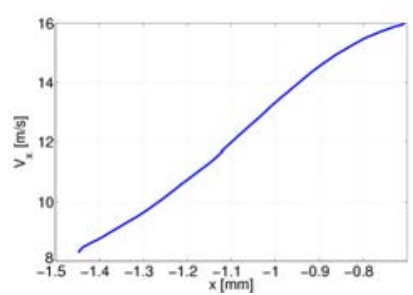

(b)
Figure 5: Location P1; a) velocity vector field and velocity magnitude, image width corresponds to about $0.7 \mathrm{~mm}$; b) extracted profile for the $x$-component of velocity along the $x$-direction; position $x=-1$ corresponds to the entrance to the gap.

The main reason of these flow acceleration and deceleration is the rapid change of the channel height in the vicinity of the processing element. The channel height is $1.5 \mathrm{~mm}$ before the gap, decreases to $0.4 \mathrm{~mm}$ in the gap, and expands to $7.5 \mathrm{~mm}$ behind the gap. Due to the fluid acceleration and deceleration strong shear stresses are produced in the flow. In case of emulsion flow, these regions of high energy dissipation are responsible for the break-up of the oil droplets.

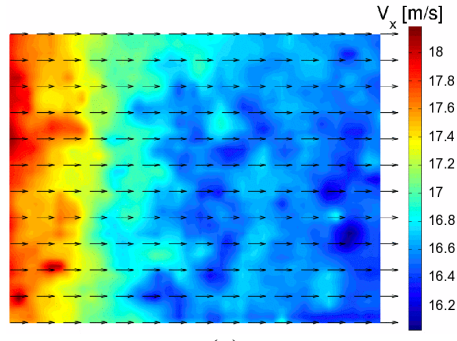

(a)

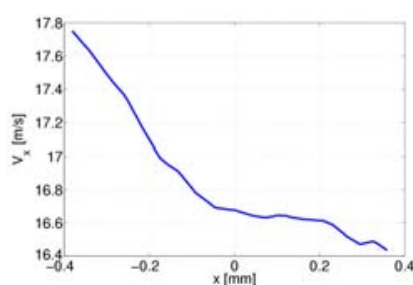

(b)
Figure 6: Location P2; a) velocity vector field and velocity magnitude (width of the image corresponds to about $0.7 \mathrm{~mm}$ ); b) extracted profile for the $x$-component of velocity along $x$-direction; position $x=-1$ corresponds to the entrance to the gap. 
Profiles of the time-averaged $x$-component of velocity along the $y$-direction (channel height) were calculated for positions P3, P4 and P5 (Fig. 7). The coordinate value $y=0$ corresponds to the surface of the upper glass wall (Fig. 1), and value $y=-3.75 \mathrm{~mm}$ corresponds to the centre of the emulsifier. $\left\langle V_{x}\right\rangle$ and $\left\langle V_{z}\right\rangle$ are the mean values of the velocity $x$-component $V_{x}$ and y-component $V_{z}$, respectively. The velocity fluctuations $V_{x}^{\prime}$ and $V_{z}^{\prime}$ are obtained from the relations:

$V_{x}=\left\langle V_{x}\right\rangle+V_{x}^{\prime}$

$V_{z}=\left\langle V_{z}\right\rangle+V_{z}^{\prime}$

The total Turbulent Kinetic Energy TKE is defined as:

$T K E=0.5\left(\left\langle V_{x}^{\prime 2}\right\rangle+\left\langle V_{y}^{\prime 2}\right\rangle+\left\langle V_{z}^{\prime 2}\right\rangle\right)$.

For two dimensional velocity measurements a modified Turbulent Kinetic Energy $\left(t e_{x z}\right)$ was defined, obtained from two available horizontal components of the velocity:

$t k e_{x z}=\left\langle V_{x}^{\prime 2}\right\rangle+\left\langle V_{z}^{\prime 2}\right\rangle$

Figure 7 shows the variation of $t k e_{x z}$ (red dashed line) calculated for selected locations P3, P4 and P5. Profiles of turbulent kinetic energy $t e_{x z}$ indicate that turbulent fluctuations emerge just behind the gap and persist along the $x$-direction. The $t e_{x z}$ reaches its maximum about $0.3 \mathrm{~mm}$ below the glass wall. This is the region, where intense mixing occurs between high speed fluid arriving from the gap and low velocity recirculating flow behind the processing element.

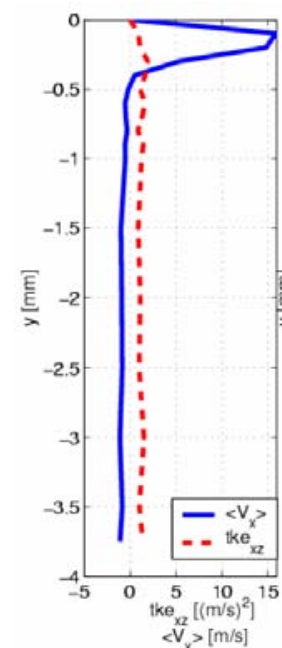

(a)

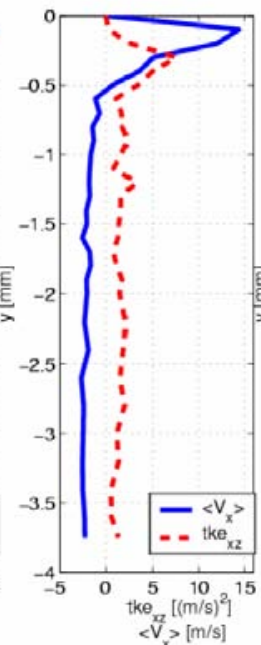

(b)

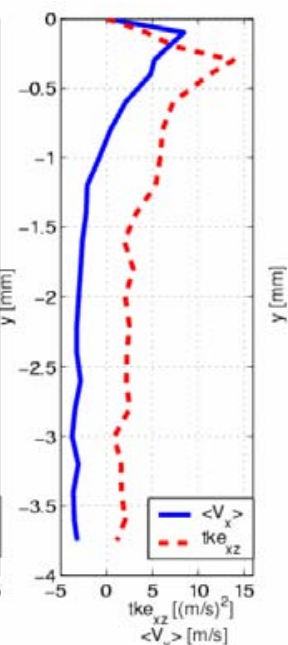

(c)
Figure 7: Time-averaged $x$-component of velocity $\left\langle V_{x}\right\rangle$ (blue solid line) and $t k e_{x z}$ (red dashed line) obtained along y-direction for locations: a) P3, b) P4 and c) P5.

In order to evaluate the persistence of the turbulence, the structure function was calculated for the flow behind the processing element. The second order transverse structure function is defined as a mean square increment of the velocity component perpendicular to the line of separation $l$. For the velocity components $V_{x}$ and $V_{z}$ it is defined as:

$S_{\mathrm{X}}^{\perp}(l)=\left\langle\left[V_{X}(x, z+l)-V_{X}(x, z)\right]^{2}\right\rangle$

$S_{\mathrm{Z}}^{\perp}(l)=\left\langle\left[V_{Z}(x+l, z)-V_{Z}(x, z)\right]^{2}\right\rangle$

The second order longitudinal structure function is defined as a mean square increment of the velocity component in the direction of two points separation. For the velocity components $V_{x}$ and $V_{z}$ and point separation $l$ it is defined as:

$S_{X}^{\|}(l)=\left\langle\left[V_{X}(x+l, z)-V_{X}(x, z)\right]^{2}\right\rangle$
$S_{Z}^{\|}(l)=\left\langle\left[V_{Z}(x, z+l)-V_{Z}(x, z)\right]^{2}\right\rangle$

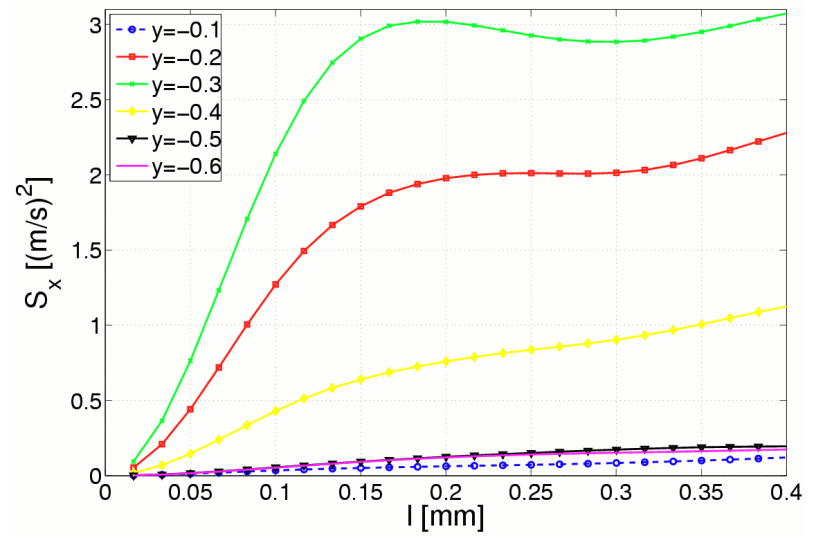

(a)

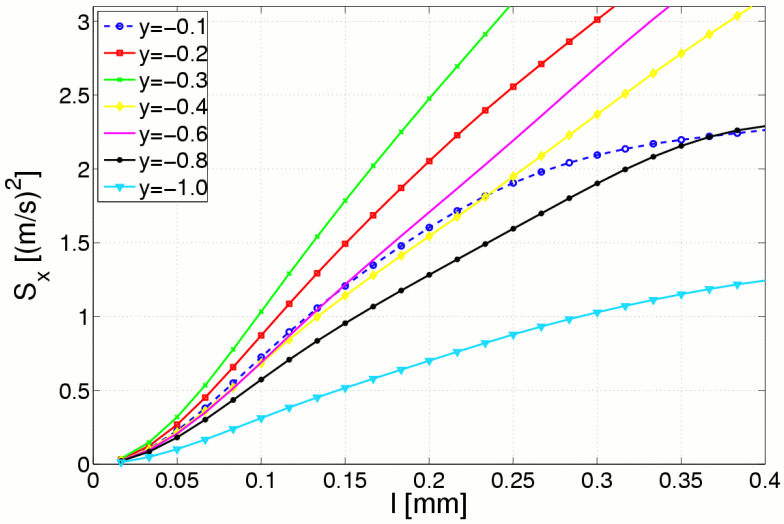

(b)

Figure 8: The second order transverse structure functions for $\boldsymbol{x}$-component of velocity $V_{X}$ evaluated for different levels $y$ at locations: a) P3 (1mm behind gap) and b) P5 (8mm behind gap).

The assumption of homogeneity of velocity field within the small area covered by our PIV measurements was used in these calculations. Mean square increments were averaged for certain separation $l$ over whole velocity field obtained from the PIV 
measurements. Then, the curves showing dependence of the structure function on the separation distance $l$ were calculated. Finally, several curves, obtained from different PIV measurements made for the same flow, for the same location, and for the same level $y$ were averaged. The results for $V_{x}$ velocity component are displayed in Fig. 8 .

Figure 8a shows transverse structure functions of $V_{x}$ velocity component, obtained for location P3, i.e. $1 \mathrm{~mm}$ behind the gap. Fast initial rising of the transverse structure function is observed for the channel depths $y=-0.2 \mathrm{~mm}$ (red line) and $y=-0.3 \mathrm{~mm}$ (green line). However, both curves flatten for larger separation length, starting already from $l>0.15 \mathrm{~mm}$. Transverse fluctuations of velocity can be used to identify development of turbulent vortices. Hence, it can be deduced that near the end of the gap small but strong vortices are dominant structures of the velocity field. Distribution of the transverse structure function for this location exhibits increased values mainly for the levels $y=-0.3 \mathrm{~mm}, y=-0.2 \mathrm{~mm}$ and $y=-0.4 \mathrm{~mm}$. It indicates that, for location $\mathrm{P} 3$, the region of high velocity increments develops between $y=-0.1 \mathrm{~mm}$ and $y=-0.5 \mathrm{~mm}$, with the maximum value at $y=-0.3 \mathrm{~mm}(0.3 \mathrm{~mm}$ below the glass window). For the location P5 ( $8 \mathrm{~mm}$ behind the gap) the maximum of velocity increments remains at the level $y=-0.3 \mathrm{~mm}$ (green line in Fig. 8b), but high velocity gradient is also relatively large at other levels. It can be deduced that there is a trail of high velocity gradients, which is thin near the end of the gap and expands along the $x$ direction.

\section{VISUALIZATION OF DROPLETS BREAK-UP}

Nearly the same flow system was used to analyse emulsification process for the very dilute oil-water mixture. For visualization of droplets break-up the modified emulsifier with a transparent processing element was used. It permitted backlight illumination of the flow in the gap between processing element and glass window. The illumination is necessary for identifying passing oil droplets.

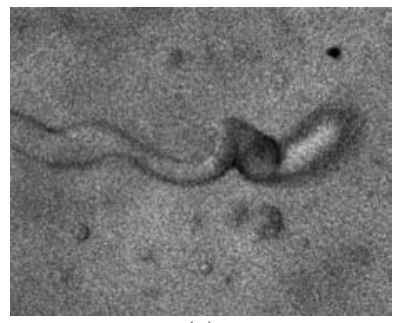

(a)

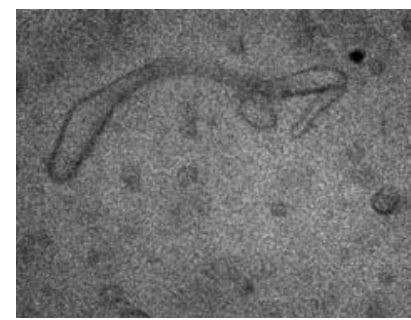

(b)
Figure 9: Deformed oil droplets observed $2 \mathrm{~mm}$ behind processing element and $0.4 \mathrm{~mm}$ below glass wall in the $\mathrm{S} 50$ silicone oil emulsion: a) without surfactant and b) with $1 \%$ wt SDS surfactant.

Emulsion was prepared using $10 \mathrm{mM} \mathrm{NaCl}$ solution in deionised water as a continuous phase. Two continuous phase systems were tested, one without surfactant and one with $1 \% \mathrm{wt}$ anionic surfactant sodium dodecyl sulphate (SDS). Two silicone oils were used as a dispersed phase: S50 ( $\mu=0.05 P a s)$ and S500 ( $\mu=0.5 \mathrm{Pas})$. All experiments were done for the flow rate $\mathrm{Q}=0.204 \mathrm{dm}^{3} / \mathrm{s}$.
The flow observation was done using Nikon Eclipse 50i epi-fluorescent microscope equipped with 20x lens and $P C O$ SensiCam camera. Double pulse Nd:YAG laser $(532 \mathrm{~nm}, 30 \mathrm{~mJ}$ per pulse) was used as a light source. Two consecutive images of emulsion were taken at the interval of $1 \mu$ s and used to evaluate instantaneous velocity magnitude of the individual droplet. Figure 9 illustrates deformation and break-up of large droplets. A few small, hardly visible droplets are also present in the view area. The image width corresponds to $432 \mu \mathrm{m}$ length of the channel.

Visualisation of droplets break-up in the emulsifier indicated that the emulsification process takes place just a few millimetres behind the processing element. However, "large" droplets were not found in the gap and droplets break-up process was not observed there. On the other hand, there are "large" droplets observed behind the gap (Fig. 9). It is not clear if these droplets are created due to the agglomeration in the dead-water region (recirculation zone behind the gap), or if it was not possible to find them in the gap using present experimental setup. It has to be mentioned that the flow observation area covers only the vicinity of the channel centre. It is possible that some large droplets were trapped by the side walls of the gap and could not be observed by the camera. These droplets may have entered the re-circulation zone and, being transported by a cross-flow to the flow axis, could appear in the observation area. It would indicate the existence of a strong cross-flow in this region. In fact, high velocity fluctuations were indicated in this region by the velocity measurements described above.

\section{NUMERICAL SIMULATION}

Numerical simulation of the flow of water was done using CFD code Fluent (Fluent Inc). Two types of numerical simulations were performed: direct unsteady simulation of laminar flow - to evaluate flow fluctuations, and $k-\varepsilon$ average turbulence model simulation - to reproduce the flow structure and turbulence characteristics in the vicinity of the processing element.

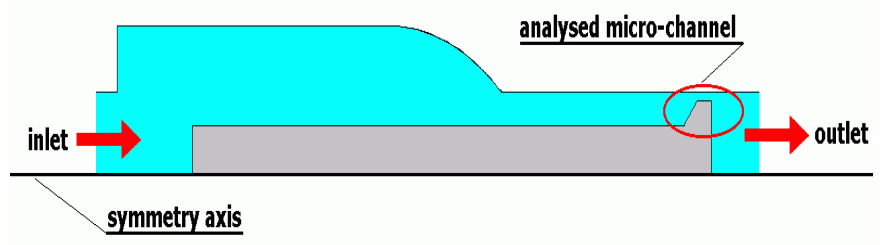

Figure 10: Schematic of geometry of the computational domain used for simulating the flow in the emulsifier.

In the first case (unsteady, laminar flow model) simulation was done for the numerical domain describing full 3D geometry of the channel. For the steady, average turbulence model, the flow symmetry was assumed and only one quarter of the model geometry was used for the computational domain (Fig. 10). Structural hexahedron mesh with boundary layer was generated in the gap and in the vicinity of the processing element. The tetrahedron mesh was used in the remaining parts of the computational domain. For the gap height 30 mesh nodes were used. Detailed parameters used for the unsteady laminar 
flow simulation and for steady turbulent flow simulation, are collected in Table 2 and 3, respectively.

Table 2: Parameters of the unsteady laminar flow simulation.

\begin{tabular}{|c|c|}
\hline $\begin{array}{l}\text { Used package and } \\
\text { version }\end{array}$ & $\begin{array}{l}\text { Fluent } 6.2 .16 \text {, double precision, } \\
\text { segregated }\end{array}$ \\
\hline Flow type & $\begin{array}{l}\text { three-dimensional, laminar, unsteady, } \\
\text { incompressible }\end{array}$ \\
\hline Medium & $\begin{array}{l}\text { water, constant density } \rho=998.2 \mathrm{~kg} / \mathrm{m}^{3} \\
\text { and viscosity } \mu=0.001003 \text { Pas }\end{array}$ \\
\hline Mass flow-rate & $\mathrm{Q}=0.204 \mathrm{~kg} / \mathrm{s}$ \\
\hline Inlet & mass-flow inlet \\
\hline Outlet & pressure outlet \\
\hline Discretization & Pressure: standard \\
\hline Time Step Size & $1 e^{-7} s$ \\
\hline Geometry and grid & $\begin{array}{l}\text { whole model geometry, } 1745830 \text { cells of } \\
\text { the grid }\end{array}$ \\
\hline
\end{tabular}

Table 3: Parameters of the steady turbulent flow simulation.

\begin{tabular}{|c|c|}
\hline $\begin{array}{l}\text { Used package and } \\
\text { version } \\
\text { Flow type }\end{array}$ & $\begin{array}{l}\text { Fluent } 6.2 .16 \text {, double precision, } \\
\text { segregated } \\
\text { three-dimensional, steady, } \\
\text { incompressible }\end{array}$ \\
\hline Viscosity model & $\begin{array}{l}\text { standard } \mathrm{k}-\varepsilon \text { turbulence model with } \\
\text { Enhanced Wall Treatment }\end{array}$ \\
\hline Medium & $\begin{array}{l}\text { water, constant density } \rho=998.2 \mathrm{~kg} / \mathrm{m}^{3} \\
\text { and viscosity } \mu=0.001003 \text { Pas }\end{array}$ \\
\hline Mass flow-rate & $\begin{array}{l}0.051 \mathrm{~kg} / \mathrm{s} \text { (one quarter of } \mathrm{Q}=0.204 \mathrm{~kg} / \mathrm{s} \text { ) } \\
\text { mass-flow inlet }\end{array}$ \\
\hline Inlet & $\begin{array}{l}\text { turbulence intensity } 12.1 \% \\
\text { hydraulic diameter } 0.0109 \\
\text { pressure outlet }\end{array}$ \\
\hline Outlet & $\begin{array}{l}\text { turbulence intensity } 12.1 \% \\
\text { hydraulic diameter } 0.0109\end{array}$ \\
\hline & $\begin{array}{l}\text { Pressure: standard } \\
\text { Momentum: Second Order Upwind }\end{array}$ \\
\hline $\begin{array}{l}\text { Discretization } \\
\text { Scheme }\end{array}$ & $\begin{array}{l}\text { Turbulence Kinetic Energy: Second Order } \\
\text { Upwind } \\
\text { Turbulence Dissipation Rate: Second } \\
\text { Order Upwind }\end{array}$ \\
\hline Geometry and grid & $\begin{array}{l}\text { one quarter of the model geometry, } \\
457473 \text { cells of the grid }\end{array}$ \\
\hline Grid Adaptation & $\begin{array}{l}\text { dynamic adaptation based on velocity } \\
\text { magnitude gradient: refine threshold } \\
0.0001 \text {, interval: } 20 \text { iterations } \\
Y+: \text { allowed value } 1-2\end{array}$ \\
\hline
\end{tabular}

Figure 11 shows velocity field obtained from laminar flow simulation averaged over 20 time steps. It can be found that before the gap the average flow velocity is about $5 \mathrm{~m} / \mathrm{s}$. In the gap strong acceleration of the flow occurs and velocity peak values reach about $23 \mathrm{~m} / \mathrm{s}$.

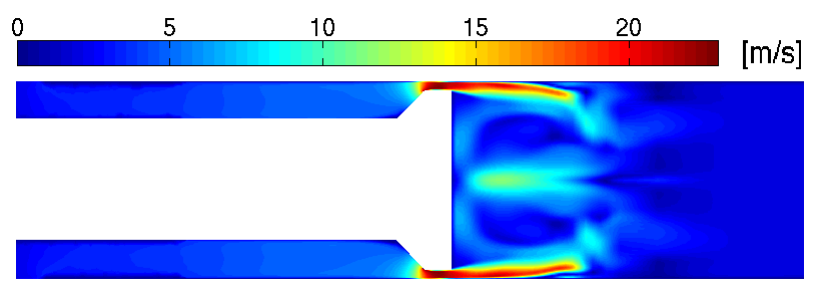

Figure 11: Numerical simulation, velocity field for laminar model.

Mean square value of the velocity fluctuations from laminar unsteady simulation, defined as previously:

$t k e_{x z}=\left\langle V_{x}^{\prime 2}\right\rangle+\left\langle V_{z}^{\prime 2}\right\rangle$,

was computed, where $V_{X}^{\prime}$ and $V_{Y}^{\prime}$ are fluctuations of the velocity components obtained from the numerical simulation. Distribution of the $t k e_{X Y}$ (Fig. 12) shows that flow turbulence, i.e. the averaged velocity fluctuations, reached their maximum value in the gap. The magnitude, amplitude and location of the velocity fluctuations found for the gap flow are in relatively good agreement with the experimental data obtained from the PIV measurements.

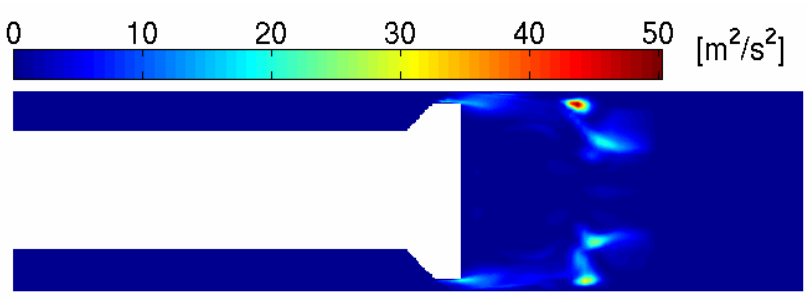

Figure 12: Numerical simulation, mean square value of the velocity fluctuations, laminar model.

Direct numerical simulations utilize large amount of the computer resources and computational time. Therefore more detailed analysis was performed using averaged turbulent model. Figures 13 and 14 show velocity field for the longitudinal section along central part of the model in the vicinity of the gap and in the region behind processing element. Velocity magnitude attains maximum value of about $20 \mathrm{~m} / \mathrm{s}$ in the gap between processing element and the top wall. It can be found that recirculation flow behind the gap region is quite significant. Velocity magnitude of the reverse flow is about $-2 \mathrm{~m} / \mathrm{s}$ (blue vectors in the Fig. 13).

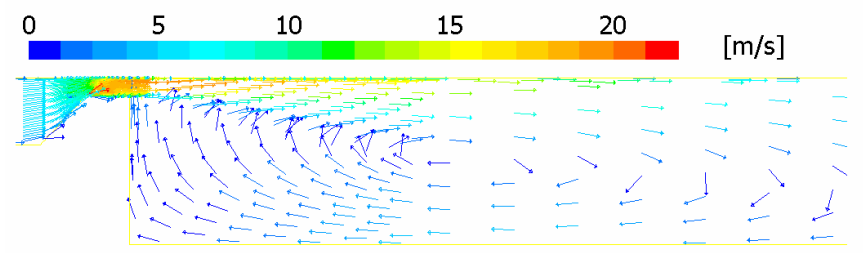

Figure 13: Velocity vectors in the vicinity of the processing element, turbulent model. 


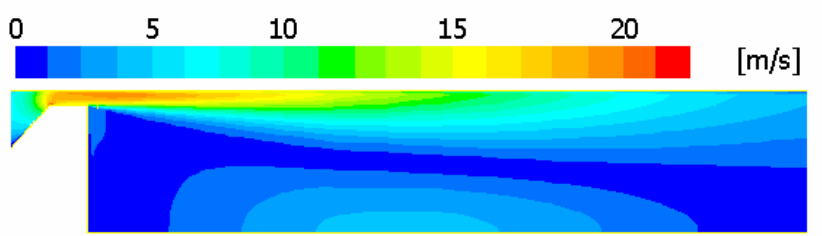

Figure 14: Contours of the velocity magnitude, turbulent model.
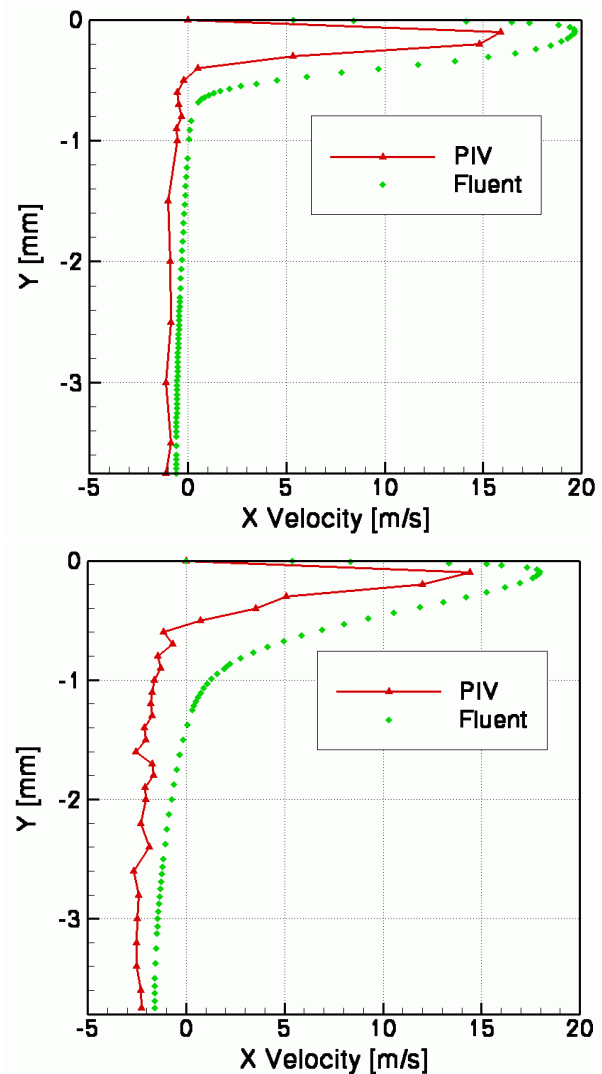

(a)

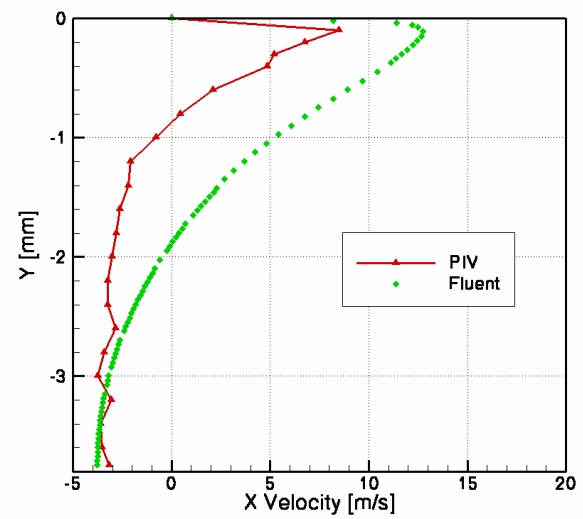

(b)

(c)

Figure 15: Comparison of numerical and experimental longitudinal velocity profiles along channel depth for three locations behind the processing element: (a) - 1mm; (b) - 3mm; (c) - 8mm.

Comparison of numerical and experimental results was done to validate numerical simulations. Figure 15 shows profiles of the $\mathrm{x}$-component of the velocity, obtained from the numerical simulations (green points) and from the PIV measurements (red line) for three locations behind the processing element: 1mm (Fig. 15a), 3mm (Fig. 15b), and 8mm (Fig. 15c).

Qualitative and quantitative agreement of the numerical and experimental results in the vicinity of the processing element is fairly good (Fig. 15a,b). For the profile located $8 \mathrm{~mm}$ behind the processing element (Fig. 15c) the difference between numerical and experimental results becomes significant, although location of the velocity maximum is still preserved. Presence of the strong reverse flow behind the gap is probably responsible for diminishing of the resolution of the PIV evaluation, which in turn decreases peak magnitude of the measured velocity vectors.

In the turbulent flow droplets break-up rate is related to the magnitude of the kinetic energy dissipation. Distribution of the turbulent kinetic energy, obtained from the numerical simulations (Fig. 16) shows that maximum value is reached in the region behind the processing element. Intense mixing of the high velocity fluid, ejected from the gap, with the low velocity recirculation flow regions behind the processing element takes place there.

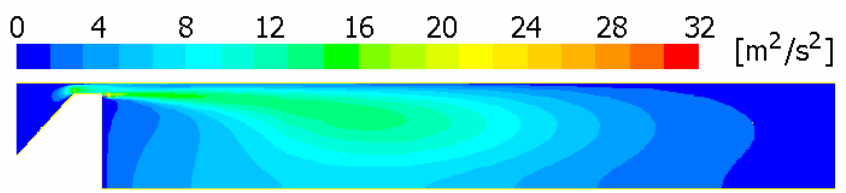

Figure 16: Contours of Turbulent Kinetic Energy.

Figure 17 shows profile of the averaged turbulent dissipation rate $\varepsilon$ evaluated along the gap. It is visible that $\varepsilon$ has sharp maximum just at the inlet of the gap. The maximum value is about $\varepsilon^{\max }=1.160 \cdot 10^{6} \mathrm{~m}^{2} / \mathrm{s}^{3}$. At the end of the gap turbulent dissipation rate rapidly decreases to the value of about $\varepsilon=1.9 \cdot 10^{5} \mathrm{~m}^{2} / \mathrm{s}^{3}$. The averaged value over the whole gap is $\varepsilon^{a v g}=3.599 \cdot 10^{5} \mathrm{~m}^{2} / \mathrm{s}^{3}$. The question arises, which value is responsible for the final size of the emulsion droplets? To elucidate this problem calculated values of $\varepsilon$ were used to test credibility of two simple models of the droplet break-up.

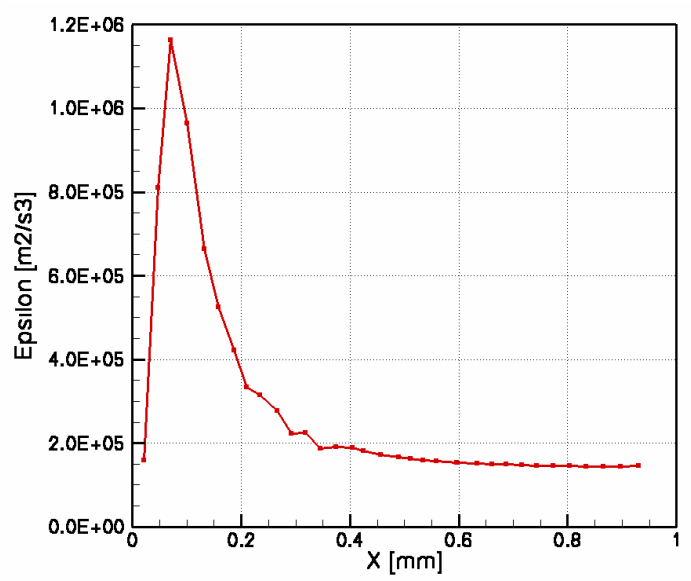

Figure 17: Horizontal profile of the averaged Turbulent Dissipation Rate $\varepsilon$ through the gap. 


\section{ESTIMATION OF THE DROPLETS MAXIMUM SIZE}

Expected oil drop size was evaluated for the investigated configuration, using estimates of the numerical simulations for the dissipation energy. Two different models of droplet breakup in turbulent flow field were tested.

- $\quad$ Hinze model [6]: it neglects viscous effects on droplet break-up process and gives following estimate of the droplet diameter:

$$
d_{\max }=0.749 \frac{\sigma^{3 / 5}}{\rho^{3 / 5} \cdot \varepsilon^{2 / 5}},
$$

where: $d_{\max }-$ maximum oil droplet diameter, $\sigma-$ interfacial tension, $\rho$ - dispersing medium density, $\varepsilon-$ turbulent dissipation rate;

- Davis [7] model, which incorporates effect of the viscosity of the dispersed phase. Here, the droplet diameter is given by:

$$
d_{\max }=\frac{K}{\rho^{3 / 5} \cdot \varepsilon^{2 / 5}}\left(\sigma+\frac{\mu \sqrt{2}\left(\varepsilon \cdot d_{\max }\right)^{1 / 3}}{4}\right)^{3 / 5},
$$

where: $d_{\max }-$ maximum oil droplet diameter, $K-$ constant $(K=0.748), \sigma-$ interfacial tension, $\rho-$ dispersing medium density, $\varepsilon$ - turbulent dissipation rate, $\mu$-dispersed phase viscosity.

Estimation was done for two silicone oils used in the emulsification experiment [1,8]: S50 with 50mPas, and S500 with ten times higher viscosity - 500mPas. The continuous phase used in the emulsification experiment [1,8] was deionised water with $1 \%$ wt anionic surfactant sodium dodecyl sulphate (SDS). The density and surface tension used in the evaluations were $\rho=998 \mathrm{~kg} / \mathrm{m}^{3}$ and $\sigma=5.5 \mathrm{mN} / \mathrm{m}$, respectively. Table 4 collects results of estimation of the oil droplets diameter, obtained applying both droplet break-up models. Calculation was done for two silicone oils and for two estimates of the turbulent dissipation rate obtained in the numerical simulations: maximum value in the gap $\varepsilon_{\max }$ and the averaged value over the whole gap $\varepsilon_{a v g}$.

Table 4: Results of the oil drops diameter estimation.

\begin{tabular}{|l|c|c|c|}
\hline & $\begin{array}{c}\text { Hinze model } \\
\text { both oils } \\
{[\mu \mathrm{m}]}\end{array}$ & $\begin{array}{c}\text { Davis model } \\
\text { oil S50 } \\
{[\mu \mathrm{m}]}\end{array}$ & $\begin{array}{c}\text { Davis model } \\
\text { oil S500 } \\
{[\mu \mathrm{m}]}\end{array}$ \\
\hline $\begin{array}{l}\text { max. epsilon value in gap } \\
\varepsilon_{\max }=1.160 \cdot 10^{6} \mathrm{~m}^{2} / \mathrm{s}^{3}\end{array}$ & 1.97 & 6.46 & 32.77 \\
\hline $\begin{array}{l}\text { avg. epsilon value in gap } \\
\varepsilon_{\mathrm{avg}}=3.599 \cdot 10^{5} \mathrm{~m}^{2} / \mathrm{s}^{3}\end{array}$ & 3.14 & 8.95 & 44.01 \\
\hline
\end{tabular}

Experiments performed at the University of Sophia [1,8] for the model emulsifier indicate droplets diameters of about $10.1 \mu \mathrm{m}$ and $20.7 \mu \mathrm{m}$ for silicone oils S50 and S500, respectively. Obviously the Hinze model overestimates the effects of turbulence on emulsification process. For the Davis model it appears that only experimental data for less viscous oil are close to the model estimates, and only if averaged value of turbulent dissipation rate $\varepsilon$ is used.

\section{CONCLUSIONS}

Velocity measurements (micro-PIV) indicated almost uniform velocity flow field in the gap region of the emulsifier model. It means that turbulence is still not fully developed and only strong shear gradients could be responsible for the droplet break-up. Strong recirculation zone with the reversal of flow is found behind the processing element. The turbulent fluctuations of the velocity field and break-up of the flow symmetry, observed in this region, indicate that probably transition from laminar to turbulent flow regime occurs there.

Numerical modelling confirmed main details of the velocity flow field measured by the micro-PIV method. It confirmed that generated numerical data can be applied for predicting conditions for droplets break-up in a shear flow. The laminar and turbulent flow models were successfully applied producing similar flow structure. It indicated that intensity of turbulence is relatively low and that the droplets break-up process may depend not only on the turbulent dissipation energy but also on the shear gradients of strongly fluctuating in time quasi-laminar flow field.

\section{ACKNOWLEDGMENTS}

This investigation was conducted in the framework of EMMA project, supported by Austrian Ministry of Science and Education, contract no.: GZ 45.534/1-VI/6a/2003 CONEX.

\section{REFERENCES}

[1] Brenn, G.; Final Scientific and Management Report of EMMA Project, (project Coordinator), Graz University of Technology, Austria 2005

[2] Raffel, M.; Willert, C.; Kompenhans, J.; Particle Image Velocimetry, A Practical Guide; Springer 1998

[3] Quenot, G.M., Pakleza, J.; Kowalewski, T.A.; Particle image velocimetry with optical flow; Exp. in Fluids 25 (1998), 177-189

[4] Stone, H.A. ; Dynamics of drop deformation and break-up in viscous fluids; Ann. Rev. Fluid Mech. 26(1994), 65-120.

[5] Blonski, S.; Kowalewski, TA.; Micro-flows investigation in production process of emulsions containing nanoparticles; CD-ROM Proceedings of Euromech Colloquium 472, Grenoble, 2005

[6] Hinze, J.O.; Fundamentals of the hydrodynamic mechanism of splitting in dispersion process; A.I.Ch.E.J. 1 (1955) 289-295.

[7] Davis, J.T.; Drop size of emulsions related to turbulent energy dissipation rates; Chem. Eng. Scs. 40 (1985) 839842.

[8] Tcholakova, S.; Denkov, N.D.; Danner, T.; Role of surfactant type and concentration for the mean drop size during emulsification in turbulent flow; Langmuir 20 (2004), 7444-7458. 